\title{
El ser humano y la génesis del trabajo enfermero comunitario
}

\section{The human being and the genesis of community nursing work}

\section{O homem ea gênese do trabalho de enfermagem comunidade}

Jorge Mínguez Arias'; José Ramón Martínez Riera²

${ }^{1}$ Doctor por la Universidad de Alicante. Enfermero Centro de salud de Arnedo. Licenciado en Antropología Social y Cultural. Profesor de Enfermería Comunitaria

${ }^{2}$ Doctor por la Universidad de Alicante.Enfermero. Master en Ciencias de la Enfermería.Vicedecano de Coordinación Académica de Enfermería. Facultad de Ciencias de la SaludCampus de San Vicent del Raspeig. Universidad de Alicante.

Cómo citar este artículo en edición digital: Mínguez Arias, J. y Martínez Riera, J.R. (2013) El ser humano y la génesis del trabajo enfermero comunitario. Cultura de los Cuidados (Edición digital) 17, 36. Disponible en: http://dx.doi.org/10.7184/cuid.2013.36.07

Correspondencia: Jorge Mínguez Arias. Escuela Universitaria de Enfermería de La Rioja. C/ Donantes de Sangre s/n 26004 Logroño. La Rioja. Correo electrónico: Jorge.minguez@eue.unirioja.es. Tel: 941261443

Recibido: 20/12/2012. Aprobado: 11/05/2013

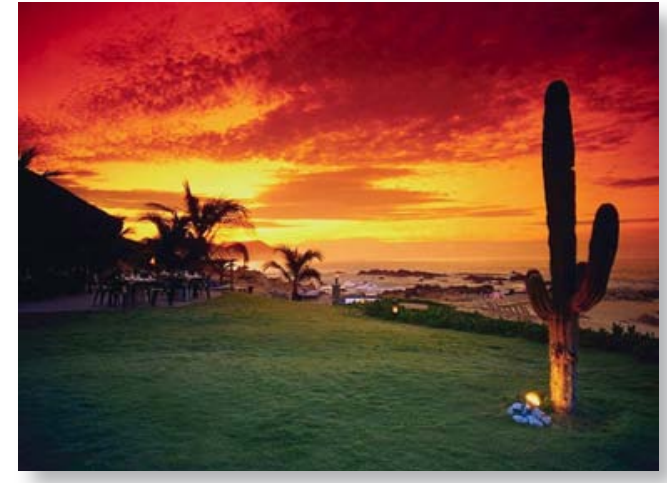

ABSTRACT

It describes the origin of nursing work and its relationship with human beings according to cultural context where it is performed. It exams terms that are socially interpreted and accepted and that are full of meanings according to Western hegemonic medical model. It also discusses the concept of man and the apparent contradiction between universality and particular diversity . Concepts, from the point of care, beyond the purely biological that allow to build a network of meanings with a psychological and social origin and continually reinvent themselves.

Keywords: Care, Labor Nurse, People, Origin nursing.

\section{RESUMO}

Ele descreve a origem do trabalho dos enfermeiros e sua relação com os seres humanos de acordo com o contexto cultural em que ele executa. Ele examina os termos são interpretados e socialmente aceites e são cheias de significados de acordo com o modelo médico hegemônico ocidental. Ele também discute o conceito de ser humano ea aparente contradição entre universalidade e diversidade particular. Conceitos que, do ponto de atendimento, além do puramente biológico e permitir-lhe construir uma rede de significados psicológicos e sociais e reinventou continuamente.

Palavras-chave: Cuidado, Enfermeiro do Trabalho, Pessoas, enfermagem Origin.

\section{RESUMEN}

Se describe el origen del trabajo enfermero y su relación con el ser humano de acuerdo al contexto cultural donde se realiza. Se examinan términos que son interpretados y aceptados socialmente y que están repletos de significados de acuerdo al modelo médico hegemónico occidental. Así mismo se analiza 
el concepto de ser humano y la aparente contradicción entre universalidad y diversidad particular. Conceptos que, desde la óptica del cuidado, trascienden lo puramente biológico y permiten construir toda una red de significados de orden psicológico y social y que se reinventan continuamente.

Palabras clave: Cuidados, Trabajo enfermero, Ser humano, Origen enfermería.

\section{INTRODUCCIÓN}

En el análisis del origen del trabajo enfermero y su relación con el ser humano se ha de examinar términos meta-paradigmáticos que frecuentemente son omnicomprensivos y por ello están repletos de importante significación mítica, que actúan como auténticos arcanos que hacen difícil su comprensión ante la falta de concreción. De ahí que sea necesario explorar, la relación particular que existe entre diferentes términos que constituyen el núcleo de la discusión (Kérouac, Pepin, Ducharme, Duquette, Major, 1995). Esto nos ayudará a reconocer el contexto cultural en el que se desarrolla, el tipo de constructo humano, sus propias connotaciones de universalidad y de diversidad particular, que a continuación se describe y analizan.

\section{DESCRIPCIÓN DEL CONTEXTO ENFER- MERO}

Para poder conocer la actividad cuidadora profesionalizada, es necesario determinar los referentes culturales y sus influencias sobre las formas de comportarse tanto los individuos como los grupos profesionales que lo conforman. Para ello es preciso describir el contexto que muestre su globalidad incluido el proceso de construcción, pues como bien indican algunos autores al referirse a salud mental "el contexto en que se ejecutan los roles tienen más influencia sobre la salud mental de las personas, que los verdaderos roles que ésta ejecuta" (Dennerstin, 1994).

Como bien indica López y Peña, la trama de las ideas donde se sustenta la enfermería comunitaria, es "la plataforma conceptual y práctica denominada salud colectiva, que reconoce los procesos de salud-enfermedadrepresentaciones-respuestas sociales, donde es necesario explicar hechos histórico-sociales que determinan los procesos más allá de la causalidad biológica” (López, Peña, 2006). Así mismo, Eseverri afirma que "la enfermería de hoy, aunque permanece enfocada hacia el futuro, paradójicamente se apoya en el pasado. Porque la enfermería siempre es de ayer, de hoy y de mañana. La enfermería de siempre y para siempre se cimienta en la historia" (Eseverri, 2006). Así es conveniente no olvidar el texto y el contexto donde ésta se desarrolla, que no es otro que el camino abierto junto con otras profesiones del ámbito de la salud, y que está en consonancia con lo que el propio Comelles indica al referirse a la consolidación corporativa de las profesiones sanitarias "ya en el siglo XVIII (la medicina entre otras), intenta buscar y asegurarse el monopolio de la gestión de la salud mediante un pacto con la burocracia estatal para excluir administrativa y jurídicamente cualquier alternativa, para recuperar para si, campos propios de los auto-cuidados, y medicalizar la sociedad basándose en la idea de universalidad que sustenta al modelo médico hegemónico" (Comelles, Doura Arnau, Martín, 1991). Esto debe de ser tenido en cuenta junto con el concepto de profesionalización, ya que hasta entonces el pensamiento prevalente era la negación de lo infinito, y a partir de ese momento se adquieren los poderes de lo positivo, pues la estructura antropológica que surge, es la de mostrar el 
rol crítico del límite, el del origen, siendo ello la esencia de la medicina positiva (Foucault, 1996). La estrategia seguida después del siglo XVIII, según el citado Comelles "se centra en dividir la atención a la salud en un sector gestionado por los médicos con criterios técnico científicos y un cajón de sastre de prácticas tradicionales o folk que deberían ser aculturadas por asistemáticas, irracionales y supersticiosas. Así la hegemonía médica se construye por parte del Estado, desde el concepto de beneficencia del Renacimiento de Luis Vives y posteriormente con el concepto de bienestar social" (Comelles, 1994). A si mismo, también hemos de añadirle algo que en el subconsciente colectivo de nuestra cultura pervive y es la representación judeo-cristiana de la idea de enfermar como castigo divino, que va unida a la tekhené griega, con la cual se ve legitimada por parte del poder político, como bien confirma Iruzubieta en su investigación sobre La Casa de Socorro de Logroño; "la higiene pública tradicional había defendido la trascendencia profiláctica de las instituciones benéficas en la medida en que partían de la concepción cristiana o caritativa" (Iruzubieta, 2006), mostrando así el arraigo histórico social en el que se sustentan.

\section{EL PROCESO DE CODIFICACIÓN SIM- BÓLICA Y SU CONSTRUCTO}

Todo ello llega a construir una red de codificaciones simbólicas, que actúan de columna vertebral que sustenta a la medicina hegemónica como algo aparentemente homogéneo, como Fdez-Rufete sostiene, "conceptos tales como biológico, natural, empírico, racional, científico, objetivo o verdadero, sustentan todo un edificio ideológico y limitativo que confiere a la medicina y a la práctica médica un estatuto ontológico que le permite reservar el monopolio de un tipo particular de violencia simbólica $y$ legítima” (Fernández-Rufete, 2000). A pesar de referirse explícitamente a la medicina, dentro de éste amplio manto conceptual, también se incluye por motivos de reflexión y análisis a la enfermería, como consecuencia del proceso de enculturación al que ha estado sometida durante años, dada su adscripción fundamentalmente al paradigma racional tecnológico. De esta forma, donde se limita a aplicar técnicas, como auxiliar subordinada a la figura del médico, siendo mera manipuladora externa de las necesidades y problemas de salud de los pacientes. Por todo esto desarrolla su labor como sub-disciplina auxiliar de otra ciencia, de la cual depende para renovar sus conocimientos técnicos, y paralelamente reproducir y ser copartícipe, por puro mimetismo de los principios que sustentan al modelo médico hegemónico occidental. Este argumento está arraigado en la sociedad, como bien mantienen De Pedro y Morales "se ha concebido tradicionalmente a la enfermera como una profesional que desempeña tareas de eminente corte práctico, para las cuales una buena formación empírica era más que suficiente. De hecho su formación siempre ha estado muy condicionada por este fenómeno, generando múltiples instituciones docentes vinculadas a hospitales que, realmente, eran centros de formación y provisión de masa laboral adiestrada a imagen y semejanza de la institución. Por tanto, el rol del conocimiento ha estado ajeno durante mucho tiempo a las enfermeras, permaneciendo incrustado en las organizaciones y en los agentes que las componen" (De Pedro, Morales, 2004). Cotidianamente se subordina la profesión enfermera frente a la profesión médica. El médico, es el referente de acción y el modelo biomédico es el camino a seguir, influidos por el imaginario colectivo en el que la entrega y dedicación es prioritaria frente a la práctica fundamentada en la cienti- 


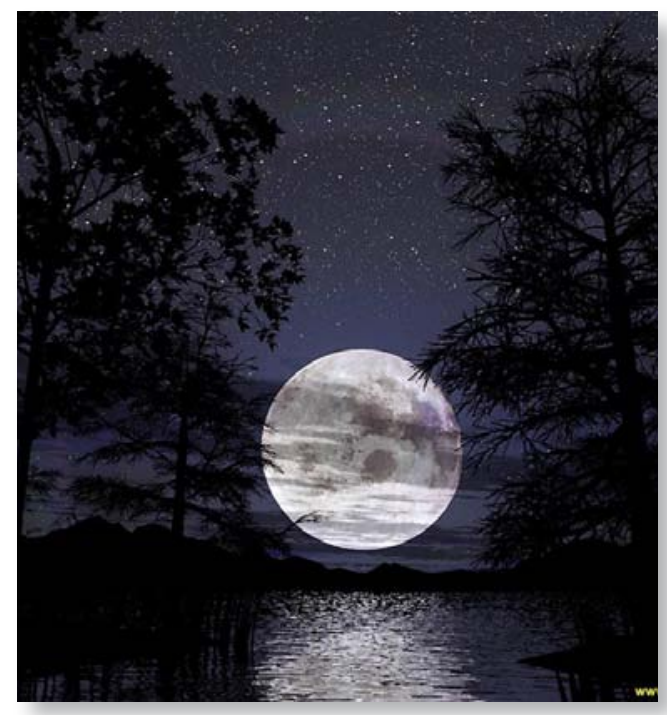

ficidad propia, donde se pueda implantar un espacio profesional con identidad enfermera (Cano, 2004).

\section{EL MODELO MÉDICO HEGEMÓNICO EN ESPAÑA}

En la España de los años sesenta, el modelo médico hegemónico, se convierte por su creciente tecnificación, en el instrumento fundamental que proporciona vida y salud en el sistema sanitario. No es coincidencia que en ese momento comiencen a surgir los primeros análisis críticos sobre el citado modelo, aunque si es cierto, que no tienen demasiada repercusión en el colectivo sanitario, a pesar de ser tan cotidianos en la actualidad, como lo muestra Ivan Illich en su obra Némesis Medica (Illich, 1974) donde acuña el término de Iatrogenesis Social, asumiendo con el citado concepto los múltiples daños que la medicalización produce (Gervás, Segura, 2007) y por tanto, como remarca Gálvez, ya se puede "escribir sobre la expropiación de la salud en la propia historia contemporánea" (Gálvez, 2002). Domingo Palacios sintetiza perfectamente estas ideas y su repercusión en la profesión, cuando afirma que "la modernidad junto con el pensamiento positivista marcaron y determinaron la manera de construir y entender el mundo entre el siglo XVII y principios del XIX. Siendo sus principales instrumentos la tecnología, la razón y la ciencia convirtieron la eficacia lo objetivo, lo pragmático, y lo tangible en elementos imprescindibles y necesarios en nuestra forma de vivir $y$ percibir nuestro entorno y nuestras relaciones" (Palacios, 2007). La enfermería como disciplina y los cuidados como objeto de estudio, estuvieron marcados por esta influencia, impregnados de este pensamiento moderno y positivista.

\section{EL SER HUMANO COMO ENTIDAD QUE SE DICE}

Existe una estrecha relación entre la historia y el ser humano, el ser humano es un ser histórico, desde que nace hasta se muere. Amparo Nogales afirma que su historia, está encadenada a la historia de la enfermería (Nogales, 2006). López mantiene que el objeto que sostiene la esencia de la actividad enfermera es el cuidado de la salud del individuo familia o comunidad. Este planteamiento tiene un amplio consenso, casi unánime entre los profesionales cualquiera que sea el cuadro paradigmático que éstos adopten "Cada corriente filosófica aborda al hombre desde una perspectiva conceptual, y fija su interés en alguna de las múltiples facetas que lo constituyen (ontología, fenomenología, cósmica..) Cada profesión, por otra parte, se acerca al hombre con una visión específica que responde al servicio que le presta $y$ a las demandas particulares que la sociedad requiere de ella" (López, 1992).

Este concepto de cuidado del ser humano es amplio y complejo, se diversifica tanto como los modelos de sistema socioculturales divergentes existen, siendo éstos, el escenario 
de referencia que condiciona su forma de ser y estar, y por tanto su comportamiento. De ahí que, para comprender el tipo de cuidado que se aplica, sea necesario analizar el concepto de ser humano desde el cual se parte.

En la historia contextual de lo humano, aparentemente existen algunos rasgos que son comunes en los distintos sistemas socioculturales, como es su constitución biológica. También existen otras características comunes que van más allá de lo puramente material, siendo este planteamiento reconocido y aceptado universalmente en todas las culturas.

\section{DESCRIPCIÓN DE LO HUMANO}

Para describir el concepto de ser humano, en principio se hará a través de esa aparente primera característica común que es su estructura morfo-biológica. Por ello, se puede afirmar que es un animal y forma parte del conjunto de los seres orgánicos vivientes, perteneciente a alguno de los linajes de los grupos biológicos, incluido en el de los grandes monos antropoides. Eso significa, que pertenece al grupo de los grandes mamíferos, dado que la hembra de la especie mantiene una relación especial con su descendencia, que es darles de mamar.

Desde este punto de vista de la realidad zoológica tiene mucha importancia ser mamífero, pues éstos, son los animales que han desarrollado comportamientos complejos de organización y de conducta, que implican una dependencia de la prole de forma prolongada en el tiempo. Este hecho tan cotidiano, la dependencia prolongada de la prole, significa que el carácter orgánico y las capacidades psicológicas con las que nace son similares a las de los seres que nacen prematuros. Todos los partos de los grandes mamíferos de acuerdo a este concepto son prematuros, esto condicio- na que tenga un largo proceso de aprendizaje, cuanto más aprendizaje, más compleja es la conducta que puede desarrollar y más complejos son los sistemas de organización en los que ajustan la vida en común de esos individuos y en el vértice de dicha complejidad está la especie humana. Esto genera un hecho que hace singular al ser humano, común en todas las culturas y que les hace diferentes al resto de las especies, y resulta que es un ser que se dice, que se cuenta, eso si, de acuerdo al escenario donde se desarrolla. Esta propiedad tan característica, nos distingue drásticamente del resto de mamíferos, pues ellos no se dicen.

El ser humano, es el animal que tiene el Don de la Palabra, con la cual se dice, se cuenta, es el animal que habla que cuenta historias. Éste reside en otro orden además del puramente biológico y es el orden narrativo, con el cual sobrevive y trasciende a la propia realidad biológica, con la que traspasa el mismo momento. Con ese otro orden permanece aunque no esté físicamente y por tanto la realidad no la vive si no la cuenta, porque así la hace comprensible, y si la comprende la posee. De ahí se puede decir que el ser humano es aquel ente biológico que habita, que está en el orden narrativo.

Esto es el hecho diferencial típicamente humano, que lo distingue de los grandes mamíferos, los monos antropoides. Constantemente se cuentan para saberse. Por eso podemos decir que el ser humano es un ente biológico psicológico y social, un todo tridimensional unificado sometido a la fuerza de la naturaleza. Capaz de reflexionar sobre los hechos colaterales y su propia experiencia, con capacidad de simbolizar y de decirse. Es un animal que sabe que tiene historia y que la dice. Se dice a través de símbolos que son elementos fundamentales para poderse comunicar (Marín, 1997). 


\section{A MODO DE CONCLUSIÓN}

Como se menciona en el comienzo en el origen del trabajo enfermero se plantean complejas situaciones que aunque omnicomprensivas necesitan ser desmenuzadas para descifrar algunos determinantes inherentes al ser humano, tales como la múltiple y compleja situación que es la comunicación, condicionada por la actitud del que trasmite, el medio utilizado para trasmitir, así como el que recibe el mensaje, todo ello arropado en un contexto que condiciona y determina la propia realidad, y presidido por la implícita capacidad de simbolizar que le asiste y guía en el vasto proceso interpretativo que realiza mediante la definición de objetos, situaciones e interpretaciones que interrelaciona lo físico con lo intelectual (Ibarra, Siles, 2006).

De ahí la importancia que tiene el considerar y conocer, desde la óptica del cuidado enfermero, la complejidad que entraña el concepto de ser humano, que va más allá de lo puramente biológico, que por su morfo-biología necesita relacionarse con los demás, lo cual permite generar toda una red muy compleja, que no habita en el orden biológico sino en el psicológico y en el social. Por eso debemos tener en cuenta, como indica Medina que "la persona es un ser bio-psico-social como un todo irreductible, en congruencia con valores y epistemologías propiamente enfermeras" (Medina, 1994), aunque la medicina hegemónica fundamente su "perspectiva analítica, empírica y experimental muy adecuada para el estudio de los procesos biológicos y biopatológicos que como fenómenos naturales que son, presentan una cierta estabilidad, regularidad, constancia y cierta independencia del contexto, pero han reducido lo humano a lo biológico" (Medina, 2006), de ahí la necesidad de seguir profundizando sobre la génesis de lo humano en el tra- bajo enfermero comunitario, dada su capacidad de auto invención de acuerdo al contexto histórico en el que se encuentra.

\section{BIBLIOGRAFÍA}

- Cano Caballero, M.D. (2004) Enfermería y género. Tiempo de reflexión para el cambio. Index Enferm. XIII (46):34-39.

- Comelles. J.M, (1994) Atención Primaria: El difícil maridaje entre atención integral y atención institucional. Jano, XLVI (1073): 79-84.

- Comelles, Doura, Arnau, Martín. (1991) L'Hospital de Valls. Asaig sobre l'estructura i les transformacions de les institucions d'asistència. Vals: Institut d'Estudis Vallencs.

- De Pedro Gómez, J.E; Morales Asensio, J.M. (2004) Las organizaciones ¿favorecen o dificultan una práctica enfermera basada en la evidencia?. Index de Enfermería; 13 (44-45):32-36. Disponible en: http://www.index-f. com/enfermería/44revista/44_articulo_26-33pht (Artículo revista)

- Dennerstein, L. (1994) Salud mental, trabajo y sexo.Quaderm CAPS; (21):62-68.

- Eseverri Chavarri, C, (2006) Hospitalidad-Enfermería, conceptos unívocos. Cultura de los cuidados. 10(20):32-38.

- Fernández-Rufete, J. (2000) El afecto perdido. En Perdiguero E. Y Comelles J.M..Medicina y cultura. Estudios entre la antropología y la medicina. Edicions Bellaterra, Bacelona: 277-303

- Foucault, M. (1996) El nacimiento de la clínica. Siglo Veintiuno de España Editores, Madrid: 277.

- Gálvez Toro, A. (2002) La medicina bajo sospecha, Seis ejercicios especulativos. Fundación Index.:16-17.

- Gervás, J; Segura Benedicto, A. (2007) Cooperación entre salud pública y atención clínica en un contexto de cambio de la utilización del sistema sanitario. Atención Primaria; 39. (6):319-322.

- Ibarra Mendoza, X; Siles González, J. (2006) Rol de enfermo crónico: Una reflexión crítica desde la perspectiva de los cuidados enfermeros. Cultura de los cuidados, 10,(20):128-13. 
- Illich, I. (1974) Némesis médica: la expropiación de la salud. Barral Editores, Barcelona.

- Iruzubieta Barragán, F.J. ( 2006) La Casa de Socorro de Logroño, 1923-1936: Origen y desarrollo. Logroño:Instituto de Estudios Riojanos:21.

- Kérouac, S; Pepin, J; Ducharme, F; Duquette, A; Major, F. (1996) El Pensamiento Enfermero. Masson, Barcelona.

- López Arellano. O, Peña Saint Martín. F, Salud y Sociedad. (2006) Aportaciones del pensamiento latinoamericano. Medicina Social. Diciembre; I (3):82-102.

- López Ruiz, J. (1992) Aproximación al concepto hombrepersona, objeto de los cuidados de enfermería. Enfermería Clínica.2 (5 ):132-36.

- Marín Pedreño, H. (1997) De Dominio Público Ensayo de teoría social y del hombre. Las Historias que somos. Pamplona: Eunsa:38-51.
- Medina Moya, J.L. (1994) Fundamentos epistemológicos de la teoría del cuidado. Enfermería Clínica. 5 (4):22132.

- Medina Moya, J.L. (2006) La complejidad del cuidado y el cuidado de la complejidad: Un tránsito pedagógico de los reduccionismos fragmentales a las lógicas no lineales de la complejidad. Texto Contexto Enferm, Florianópolis, 15(2):312-9.

- Nogales Espert, A. (2006) Pensamiento enfermero e historia. Necesidad de vertebración filosófica e historia en enfermería. Cultura de los Cuidados. $2^{\circ}$ Semestre X. (20):14-20.

- Palacios Ceña. D. (2007) La Construcción Moderna de la Enfermería. Cultura de los cuidados. 20 Semestre;11(22):26-32.

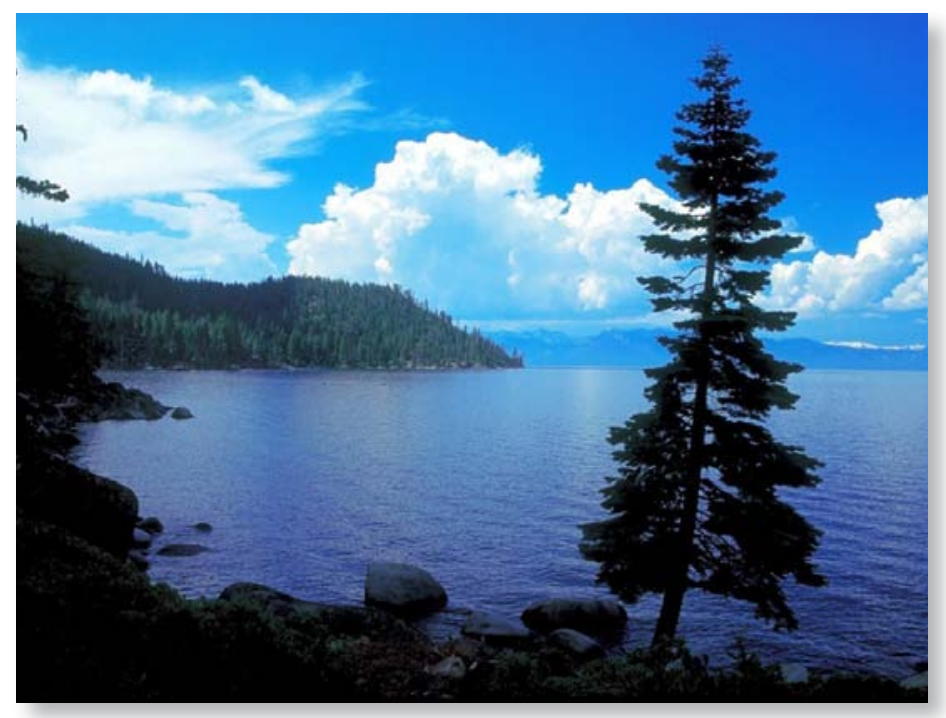

